\title{
Development of the human adrenal zona reticularis: morphometric and immunohistochemical studies from birth to adolescence
}

\author{
Xiao-Gang Hui ${ }^{1,3}$, Jun-ichi Akahira ${ }^{1}$, Takashi Suzuki ${ }^{1}$, Masaki Nio ${ }^{3}$, Yasuhiro Nakamura ${ }^{1,2}$, \\ Hiroyoshi Suzuki ${ }^{4}$, William E Rainey ${ }^{2}$ and Hironobu Sasano ${ }^{1}$ \\ ${ }^{1}$ Department of Pathology, Tohoku University School of Medicine, 2-1 Seiryo-machi, Aoba-ku, Sendai 980-8575, Japan \\ ${ }^{2}$ Department of Physiology, Medical College of Georgia, Augusta, Georgia 30912, USA \\ ${ }^{3}$ Department of Pediatric Surgery, Tohoku University School of Medicine, Sendai 980-8575, Japan \\ ${ }^{4}$ Department of Pathology and Laboratory Medicine, National Hospital Organization, Sendai Medical Center, Sendai 983-8520, Japan \\ (Correspondence should be addressed to H Sasano; Email: hsasano@patholo2.med.tohoku.ac.jp)
}

\begin{abstract}
Age-related morphologic development of human adrenal zona reticularis (ZR) has not been well examined. Therefore, in this study, 44 human young adrenal autopsy specimens retrieved from large archival files $(n=252)$ were examined for immunohistochemical and morphometric analyses. Results demonstrated that ZR became discernible around 4 years of age, and both thickness and ratio per total cortex of ZR increased in an age-dependent fashion thereafter, although there was no significant increment in total thickness of developing adrenal cortex. We further evaluated immunoreactivity of both KI67 and BCL2 in order to clarify the equilibrium between cell proliferation and apoptosis in the homeostasis of developing human adrenals. Results demonstrated that proliferative adrenocortical cells were predominantly detected in the zona glomerulosa and partly
\end{abstract}

in outer zona fasciculata (ZF) before 4 years of age and in ZR after 4 years of age, but the number of these cells markedly decreased around 20 years of age. The number of BCL2positive cells increased in $\mathrm{ZR}$ and decreased in $\mathrm{ZF}$ during development. Adrenal androgen synthesizing type $517 \beta-$ hydroxysteroid dehydrogenase (HSD17B5 or AKR1C3 as listed in the Hugo Database) was almost confined to ZR of human adrenals throughout development. HSD17B5 immunoreactivity in ZR became discernible and increased from around 9 years of age. Results of our present study support the theory of age-dependent adrenocortical cell migration and also indicated that ZR development is not only associated with adrenarche, but may play important roles in an initiation of puberty.

Journal of Endocrinology (2009) 203, 241-252

\section{Introduction}

Human adrenarche is generally characterized by the appearance of axillary hair, pubarche, and a transient acceleration of linear growth and bone maturation. Adrenarche has also been defined as the increased production of dehydroepiandrosterone (DHEA) and DHEA-sulfate (DHEAS) in human adrenal cortex, which occurs between 6 and 8 years of age (Parker \& Odell 1980, Voutilainen et al. 1983). The onset and characteristics of adrenarche are also well known to be associated with alterations in both adrenocortical functions and morphology (Parker et al. 1983, Gell et al. 1996). The zona reticularis (ZR) is generally considered the site for DHEA and DHEAS synthesis (Deperetti \& Forest 1976, Hyatt et al. 1983, Endoh et al. 1996, Rainey \& Nakamura 2008). Therefore, an increment of adrenal androgen production has been considered to be closely associated with temporal and spatial morphologic changes in the ZR during adrenarche (Dhom 1973, Parker et al. 1983, Suzuki et al. 2000, Rainey et al. 2002).
Functionally, the ZR-specific or preferential steroid metabolizing enzymes have been proposed to play important roles in the biosynthesis of DHEA and DHEAS (Suzuki et al. 2000). Dhom was the first to carefully examine the appearance and continuous development of ZR and suggested the possible correlation between adrenal androgen productions and morphologic development of the ZR in human adrenal glands (Dhom 1973). Results of other published histomorphologic studies demonstrated a reduction in histologically identifiable ZR width, which is proportional to the width of cytochrome b5 immunopositive cortical cells. (Dharia et al. 2005); however, the total cortical width remained the same throughout the ages, suggesting that ZR regression in elderly population may account for the diminished production of DHEAS, a phenomenon also termed 'adrenopause' (Parker et al. 1997). In our present study, we used immunohistochemical analysis for adrenocortical steroidogenic enzymes in order to fully characterize the morphometric and functional changes of human adrenocortical ZR in both males and females from infancy to adolescence. 
Adrenal tissue represents a cell renewal system in which the adaptive structural remodeling is accomplished by a balance between cell proliferation and apoptosis (Leblond 1964). However, the cellular dynamics underlying postnatal development of the ZR during adrenarche has remained unknown. Therefore, we further evaluated alterations of immunoreactivity of KI67 (listed as MKI67 in the Hugo Database) and BCL2 in human young adrenal cortex during $\mathrm{ZR}$ development in order to obtain a better understanding of cytogenesis of ZR.

The correlation of adrenarche with the initiation of puberty has also not been well evaluated. In particular, the morphologic correlation between adrenarche and puberty has remained still unknown. Therefore, we evaluated $17 \beta$ hydroxysteroid dehydrogenase (HSD17B5) during ZR development with attempt to study the relationship between alterations within the adrenal during adrenarche and the age of puberty. Results of a recent study have demonstrated that HSD17B5, which plays an important role in the conversion of sex steroids, is also expressed in human adrenal gland (Petry et al. 2007). HSD17B3 provides $~ 50 \%$ of the total amount of testosterone in men by synthesizing testosterone from androstenedione (Geissler et al. 1994, Labrie et al. 2005), but the same enzymatic reaction is catalyzed in peripheral target tissues via HSD17B5 and HSD17B1, not through HSD17B3. HSD17B5 has been reported to be most abundantly expressed in the human adrenal compared to HSD17B1 and HSD17B3 (Dufort et al. 1999, Qin \& Rosenfield 2000, Nakamura et al. 2009). In addition, HSD17B5 has also recently been reported to be expressed at higher levels in human ZR using quantitative PCR and immunohistochemistry (Nakamura et al. 2009). Therefore, in this study, we included the chronological analysis of HSD17B5 expression during ZR development in human adrenals using immunohistochemistry.

\section{Materials and Methods}

\section{Tissues and age groups}

Human adrenal autopsy specimens from birth to adolescence ( $n=252$, from postnatal 7 months to 20 years of age) were retrieved from autopsy files of Tohoku University Hospital and National Hospital Organization, Sendai Medical Center from 1990 to 2007 (Sendai, Japan). Forty-four specimens were selected for this study from the large group of archival tissue specimens following careful histological or morphological screening from the standpoints of the following four criteria: tissue collection in $<3 \mathrm{~h}$ postmortem; no histories of administration of adrenocortical steroids or chronic illness prior to death; no pathological abnormalities including adrenocortical nodules or neoplasms; full thickness of the adrenal extending from capsule to medulla available in the specimens. After gender stratification, the specimens were tentatively assigned into three age groups: group $0-3$ years of age $(n=5$ in male; $n=4$ in female); group $4-12$ years of age
( $n=8$ in male; $n=5$ in female); group $13-20$ years of age $(n=12$ in male; $n=10$ in female). From these paraffinembedded specimens, $3 \mu \mathrm{m}$ thickness tissue sections were prepared for immunostaining. This protocol was approved by Institutional Review Board of Tohoku University School of Medicine (2004-355) and National Hospital Organization, Sendai Medical Center.

\section{Antibodies}

The polyclonal antibodies for steroid sulfotransferase type 2A1 (SULT2A1) (1:1000, Santa Cruz Biotechnology, Santa Cruz, CA, USA, sc-18725), HSD3B2 (1:2500, rabbit antiplacental HSD3B2) employed in this study, have been previously described. Briefly, the antibody directed against HSD3B2 has been characterized as specific for immunohistochemical and western analyses (Doody et al. 1990, Lorence et al. 1990, Sasano et al. 1990). The antibody SULT2A1 was a goat polyclonal $\operatorname{IgG}$ preparation against a peptide mapping within an internal region of SULT2A1 of human origin, and has been demonstrated to be specifically expressed in human liver and adrenal tissues using both western blot and immunohistochemical evaluations (Otterness et al. 1992, Comer et al. 1993, Forbes et al. 1995). In addition, the monoclonal antibodies KI67 (MIB-1, 1:100 DAKO, Glostrup, Denmark) and BCL2 (1:80 DAKO) were also used in this study. A monoclonal anti-human HSD17B5 antibody (also called AKR1C3, 1:200 Sigma) was obtained from Sigma-Aldrich. This antibody was previously reported not to cross react with human AKR1C1, AKR1C2, or AKR1C4 (Lin et al. 2004).

\section{Immunohistochemistry}

Immunohistochemical analyses were performed by the streptavidin-biotin amplification method using a Histofine kit (Nichirei, Tokyo, Japan). Briefly, after deparaffinization, tissue sections were treated with $0 \cdot 3 \%$ hydrogen peroxidase in methanol for $30 \mathrm{~min}$ at room temperature to quench endogenous peroxidase activity and then treated with 1\% normal rabbit or goat (when the primary antibody was developed in rabbit) serum for $30 \mathrm{~min}$ at room temperature in a moisture chamber. The sections for KI67 and BCL2 immunostaining were immersed in citric acid buffer $(0.002 \mathrm{M}$ citric acid and $0.009 \mathrm{M}$ trisodium citrate dihydrate, $\mathrm{pH}$ 6.0) and heated in autoclave for $5 \mathrm{~min}$ at $127^{\circ} \mathrm{C}$ and were then allowed to cool for $\sim 1 \mathrm{~h}$ at room temperature for antigen retrieval. The diluted primary antibodies were incubated with sections for at least $18 \mathrm{~h}$ at $4{ }^{\circ} \mathrm{C}$. After rinsing in $0 \cdot 01 \mathrm{M}$ PBS, sections were incubated with the biotinylated second antibodies for $30 \mathrm{~min}$ at room temperature, followed by exposure to peroxidase-conjugated streptavidin for $30 \mathrm{~min}$ at room temperature. The antigenantibody complexes were subsequently visualized by immersion in 3,3'-diaminobenzidine (DAB) solution (0.001 M DAB, 0.05 M Tris-HCl buffer, pH 7.6, 0.01 M sodium azide, and $0.006 \%$ hydrogen peroxidase). 
Hematoxylin was used as a nuclear counterstain. We performed immunohistochemical evaluation in paraffinembedded human tissues, which are considered to serve as positive controls in order to further validate the characteristics of the antibodies employed. Briefly, human liver tissue was used for antibody SULT2A1 (Narasaka et al. 2000), Cushing's adrenocortical adenoma tissue was used for HSD3B2 (Magrini et al. 1969, Yanase et al. 1998), and tonsil was used for both KI67 and BCL2 (Martinez-Valdez et al. 1996). As negative controls of immunostaining, the primary antibodies were omitted and replaced with nonimmune serum and then the immunostaining procedure was completed as outlined above. There was no immunostaining evident in such sections.

The immunostaining procedures were all performed as carefully as possible using the same conditions for all the tissue sections.

\section{Quantitative measurement of adrenal cortex}

Quantitative analysis of adrenal sections was performed using the microscope-attached computerized image capture and analysis system (Penguin/Pro 600ES Pixel Digital Camera System, USA). First, a standard micrograph was prepared as a measurement scale with a minimum unit of $10 \mu \mathrm{m}$ using a $4 \times$ microscope objective. Micrograph of sections processed for SULT2A1 and HSD3B2 immunohistochemistry was then captured under the same conditions as the scale. Digital images of each adrenal section designating five separate quadrangle-shaped areas that contained full-thickness cross section of the cortex were prepared. Image-editing software (Adobe Photoshop Elements 5.0) was then used to adjust this captured micrograph. A straight line was drawn from the subcapsular area of the adrenal to the edge of medulla inward in the direction of cord-like zona fasciculata (ZF), followed by drawing another parallel line representative of the thickness of ZR. After these two straight lines were copied to the scale micrograph respectively, their lengths were expressed accurately in micrometers. Finally, five values were measured for each section and the average value was determined for the final analysis. To avoid any bias, all measurements were conducted in the blind fashion or without the knowledge of the age and sex of the donors. The data for analysis included the total cortical thickness, the thickness of $\mathrm{ZR}$, and the ratio of the thickness of ZR to that of the total cortex expressed by $\%(R)$.

In addition, it is difficult or subjective to identify the $\mathrm{ZR}$ using hematoxylin and eosin staining only; therefore, we performed immunohistochemistry for SULT2A1, the enzyme responsible for the sulfonation of adrenally derived DHEA that is nearly exclusively localized in the $\mathrm{ZR}$ of human adrenal cortex (Suzuki et al. 2000). Results of previous studies demonstrated that cytochrome b5 could be served as ZR-specific marker by immunohistochemistry in the adrenal cortex of the mature rhesus macaque (Mapes et al. 1999) and human (Yanase et al. 1998), but it is also true that SULT2A1 was predominantly expressed in the cytoplasm of adrenocortical cells in the ZR (Suzuki et al. 2000). In addition, similar patterns of age-related alterations of immunoreactivity between cytochrome b5 and SULT2A1 in the ZR of the human adrenal cortex have been demonstrated (Suzuki et al. 2000). In our present study, in order to clarify the border between ZF and ZR, we additionally used immunohistochemistry for HSD3B2 for tissue sections obtained from subjects older than 8 years of age when the immunoreactivity of HSD3B2 was reported to be significantly weaker in the ZR than in the ZF (Gell et al. 1998, Suzuki et al. 2000). In addition, Dardis et al. (1999) reported a decrement of mRNA levels of $H S D 3 B 2$ in adrenals from children 8 years of age and older compared to children younger than 8 years of age. The combined use of immunohistochemistry for both SULT2A1 and HSD3B2 allowed exact localization and measurement of $\mathrm{ZR}$ for the specimens obtained from the subjects older than 8 years of age. Because the ZR in the sections between 4 and 8 years of age was demonstrated to be clearly identified by SULT2A1 solely, no immunohistochemistry for HSD3B2 was supplemented in such sections.
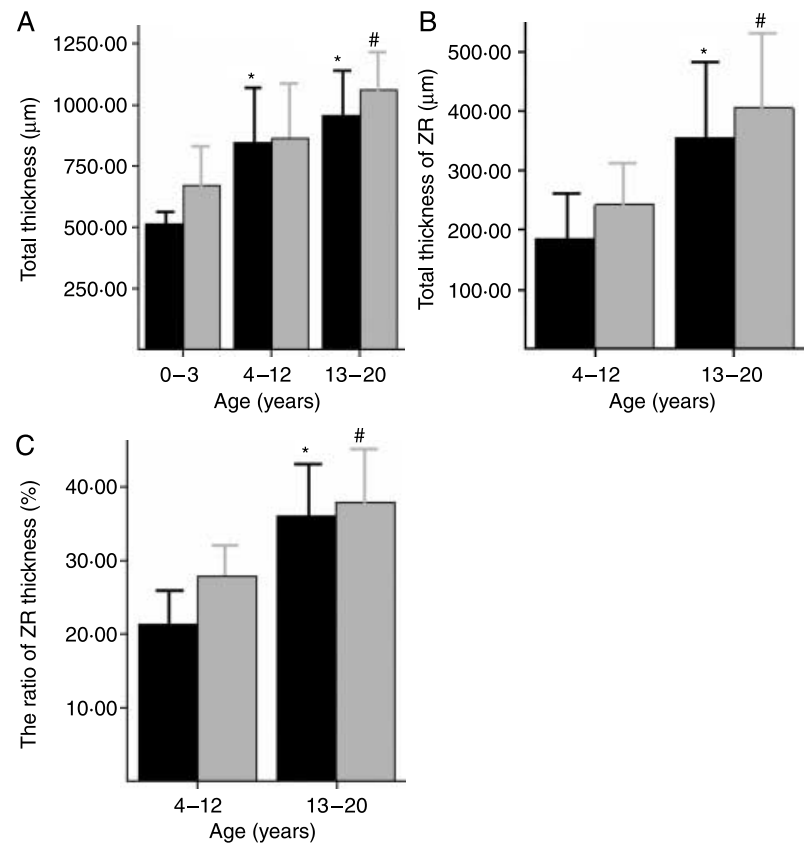

Figure 1 Quantitative analyses of age-related morphologic changes in human adrenal cortex. Adrenal cortex was studied in 25 male and 19 female adrenal glands from 0 to 20 years of age. Tissues were processed as described in Materials and Methods, and slides were photographed at $4 \times$ microscope objective. (A) Total thickness in three age categories in both male and female adrenals (* versus $0-3$ year olds in male, $P<0 \cdot 05 ;{ }^{*}$ versus $0-3$ year olds in female, $P<0 \cdot 01$ ); (B) ZR thickness in both male and female adrenals displayed more significant development of ZR in 13-20 year olds than that in the 4-12-year-old group (* versus 4-12 year olds in male, $P<0 \cdot 01$; ${ }^{\#}$ versus $4-12$ year olds in female, $P<0 \cdot 01$ ); (C) the ratio of ZR thickness to that of total cortex in both male and female adrenals showed the significant difference between $13-20$ and $4-12$ year olds (* versus $4-12$ year olds in male, $P<0 \cdot 01$; ${ }^{*}$ versus $4-12$ year olds in female, $P<0 \cdot 01)$. Black bar, male; gray bar, female. 

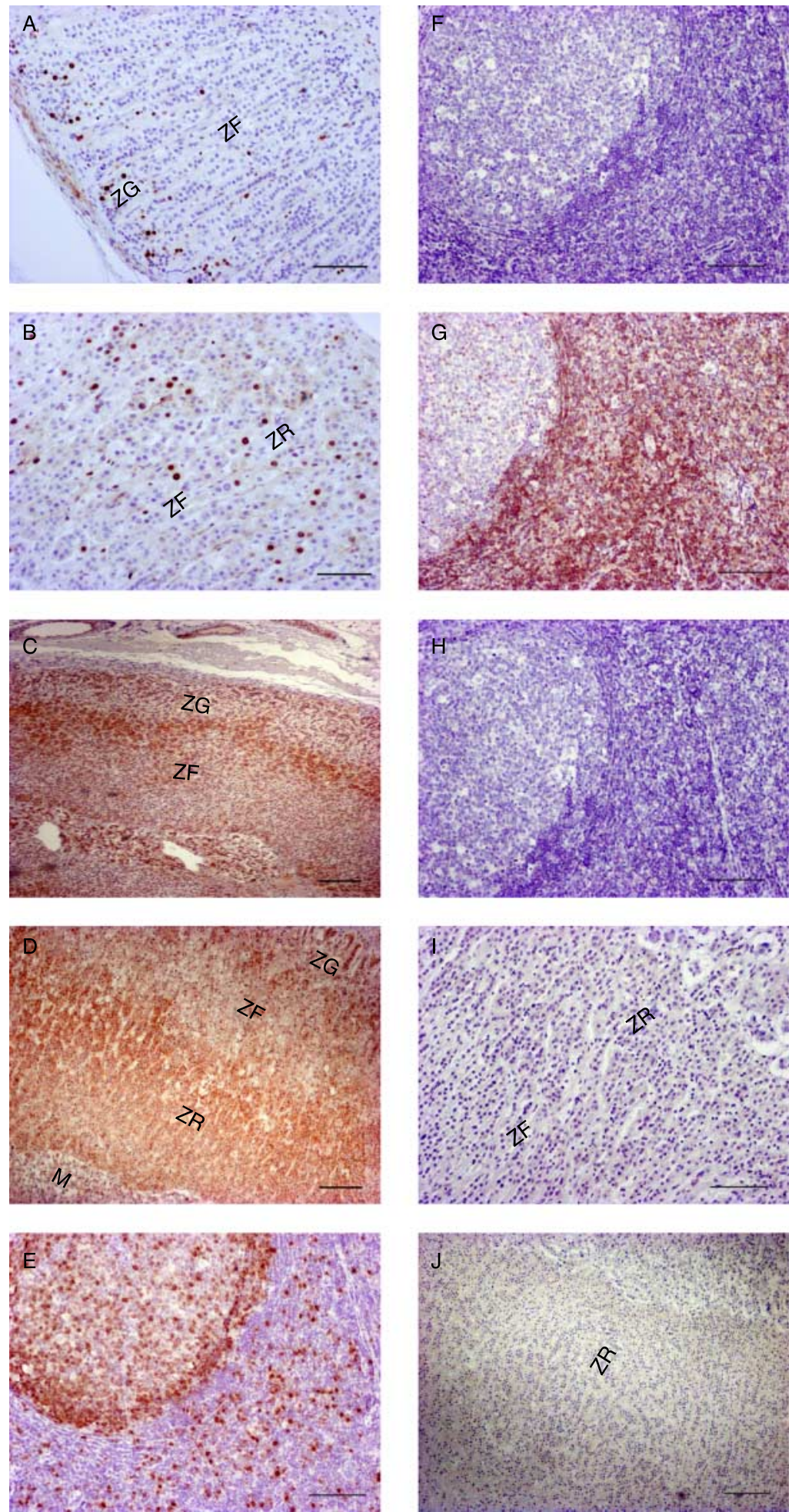
Table 1 Labeling index of KI67 among age groups

Labeling index $(\%)$

\begin{tabular}{|c|c|c|}
\hline & \\
\hline & Male (M) & Female $(F)$ \\
\hline \multicolumn{3}{|l|}{$\begin{array}{l}\text { Ages (cases of } \\
\text { specimens } M / F \text { ) }\end{array}$} \\
\hline $0-3$ years $(5 / 4)$ & $5 \cdot 22 \pm 1 \cdot 19^{*}$ & $7 \cdot 05 \pm 3 \cdot 10^{*}$ \\
\hline $4-12$ years $(8 / 5)$ & $5 \cdot 63 \pm 3 \cdot 40^{*,+}$ & $2 \cdot 50 \pm 1 \cdot 44^{*}$ \\
\hline $13-20$ years $(12 / 10)$ & $2 \cdot 64 \pm 1 \cdot 50$ & $4 \cdot 68 \pm 3 \cdot 62$ \\
\hline
\end{tabular}

$* P<0 \cdot 05$ versus age $13-20$ years within the same gender by Kruskal-Wallis rank sum test; ${ }^{\dagger} P<0.05$ versus age $4-12$ years in female by Wilcoxon rank sum test.

\section{Evaluation of immunoreactivity}

In our present study, three different methods were used to assess immunoreactivity. The immunoreactivity of KI67 was evaluated quantitatively by labeling index (LI), which represented the number of positive cells per 1000 adrenocortical cells. In detail, before counting, the areas for the analysis were determined by two of the authors (Suzuki \& Akahira ) using a double-headed light microscope. For each section, five to 10 high-power fields $(400 \times)$ were selected, and at least 1000 cells were independently counted by the two of the authors above. The tissue sections with interobserver differences of $>5 \%$ were revaluated. We calculated the mean values only in the specimens with interobserver differences of $<5 \%$. The other evaluations were performed by two of the authors (Akahira \& Hui). With respect to BCL2, a semiquantitative evaluation was used. For each adrenal section, the most immunointensitive region $(76-100 \%)$ was given an arbitrary ranking of five and the medulla, which was negative, was designated $0(\leq 1 \%)$, and a scoring of $1-4$ corresponded to $1-10,11-30,31-50$, and $51-75 \%$ respectively. Histological identification of the three zones of the adrenal cortex was based on that of previous reports (McNicol 1992). The immunoreactivity for each zone was subsequently determined by blind ranking of each slide by the authors above, and numerical values from each observer were then averaged. The quantitative evaluation of HSD17B5 was achieved by measuring the thickness of HSD17B5 immunoreactive ZR using the same measurement described above. The identical areas for both the measurements were designated in advance. The correlation between the thickness of HSD17B5 immunoreactive $\mathrm{ZR}$ and that of morphologically determined $\mathrm{ZR}$ above was estimated by regression analysis.

\section{Statistical analysis}

All data were expressed as mean \pm s.D. Statistical analyses were appropriately performed by unpaired Student's $t$-test or oneway ANOVA or Wilcoxon's test followed by Bonferroni test for comparison between two groups according to the data types. $P<0.05$ was considered significant. Spearman correlation analysis $(r)$ was used to evaluate association between two variations. All the above analyses were performed by use of SPSS software (SPSS 11.0, Chicago, IL, USA).

\section{Results}

\section{Adrenal cortex total thickness}

In the male adrenal cortex, the total thickness $(\mu \mathrm{m})$ in the 0-3-year-old group $(512 \cdot 00 \pm 49 \cdot 19)$ was significantly lesser than that in older groups examined in this study $(P<0 \cdot 01)$, whereas there was no significant difference between older groups 4-12 years of age $(846 \cdot 75 \pm 222 \cdot 43)$ and $13-20$ years of age $(956 \pm 185 \cdot 21)$. In the female adrenal cortex, no significant difference was detected between the 0-3-year-old $(669 \cdot 25 \pm 160 \cdot 46)$ and $4-12$-year-old groups $(865 \cdot 20$ $\pm 221 \cdot 48)$. However, the size of the cortex in adrenals from the 13-20-year-old group $(1063 \cdot 60 \pm 155 \cdot 74)$ was significantly larger than the $0-3$-year-old group $(P<0 \cdot 01)$ but not in the 4-12-year-old group (Fig. 1A). In male adrenals, the total thickness demonstrated a marked increment from 4 years of age until around 12 years of age but reached a plateau in the 13-20-year-old group. In female adrenals, the total thickness increased from the 0-3-year-old group and reached a plateau around 16 or 17 years of age.

\section{The thickness of the $Z R$}

The morphologically continuous ZR was not discernible in adrenals younger than 3 years of age. Therefore, the thickness of the $\mathrm{ZR}$ in the $0-3$-year-old group was recorded as zero in both the male and female adrenals. Starting from around 4 years of age, the innermost ZR became morphologically discernible and demonstrated a progressive increment with age. The thickness of the ZR in the 13-20-year-old group was significantly greater than that in the 4-12-year-old group in both male and female adrenals $(P<0 \cdot 01)$. In detail, the thickness $(\mu \mathrm{m})$ in the 4-12-year-old group was $185 \cdot 25$

Figure 2 Immunohistochemistry for KI67 and BCL2 in human adrenals. Adrenal expression of KI67 and BCL2 was studied in 44 adrenal glands from 0 to 20 years of age respectively. As shown in these representative micrographs, immunoreactivity of KI67 was almost detected in the ZG at 7 months of age (A), and strong immunoreactivity of KI67 was mostly detected in the ZR besides slight distribution in the transitional area between the ZF and the ZR around 15 years of age. (B) Immunoreactivity of BCL2 was easily detected in the outer ZF at 3 years of age (C), and strong immunoreactivity was detected in the ZR besides the weak immunoreactivity in outer ZF at 16 years of age. (D) Immunohistochemical detection of KI67 and BCL2 in human tonsil paraffin-embedded sections as positive controls were illustrated in micrograph E and $\mathrm{G}$ respectively, with corresponding negative controls in micrograph $\mathrm{F}$ and $\mathrm{H}$ respectively. The negative controls of human adrenal glands for immunohistochemistry of KI67 and BCL2 were also illustrated in micrograph I and J respectively. Bar represents $100 \mu \mathrm{m}(\mathrm{A}, \mathrm{B}$ and E-I); bar represents $200 \mu \mathrm{m}$ (C, D, and J). ZG, zona glomerulosa; ZF, zona fasciculata; ZR, zona reticularis; M, medulla. Full colour version of this figure available via http://dx.doi.org/10.1677/JOE-09-0127. 
Table 2 Summary of expression of BCL2 in human developing adrenal cortex

\begin{tabular}{|c|c|c|c|c|c|c|}
\hline & \multicolumn{2}{|l|}{ ZG } & \multicolumn{2}{|l|}{$\mathbf{Z F}$} & \multicolumn{2}{|l|}{ ZR } \\
\hline & M & $\mathrm{F}$ & M & $\mathrm{F}$ & M & $\mathrm{F}$ \\
\hline \multicolumn{7}{|l|}{ Age (cases) } \\
\hline $4-12$ years $(n=13)$ & $0 \cdot 5 \pm 0 \cdot 21$ & $0.6 \pm 0.34$ & $3 \cdot 3 \pm 0 \cdot 60^{*}$ & $3 \cdot 0 \pm 0 \cdot 44^{\dagger}$ & $2 \cdot 0 \pm 0 \cdot 83$ & $1 \cdot 5 \pm 0.54$ \\
\hline $13-20$ years $(n=22)$ & $0 \cdot 8 \pm 0 \cdot 45$ & $0.8 \pm 0.62$ & $1 \cdot 4 \pm 0.55^{*}$ & $1.5 \pm 0.62^{\dagger}$ & $4 \cdot 6 \pm 0 \cdot 43$ & $4 \cdot 3 \pm 0 \cdot 71$ \\
\hline
\end{tabular}

Arbitrary units: 5 (highest degree of staining) to 0 (negative staining). ZR was compared with ZF within each age group (except for the 0-3-year-old group). $M$, male; $F$, female; $Z G$, zona glomerulosa; $Z F$, zona fasciculata; $Z R$, zona reticularis. ${ }^{*} P<0 \cdot 05$ versus $Z R$ in male adrenals; ${ }^{+} P<0 \cdot 05$ versus $Z R$ in female adrenals.

$\pm 77 \cdot 80$ in male and $243 \cdot 00 \pm 69 \cdot 89$ in female; the thickness $(\mu \mathrm{m})$ in the $13-20$-year-old group was $356 \cdot 17 \pm 125 \cdot 26$ in male and $405 \cdot 00 \pm 126 \cdot 31$ in female (Fig. 1B).

The ratio of the thickness of $Z R$ to that of the total cortex ( $\mathrm{R}$ ) In male adrenals, the $R$ value (ZR \% of total cortical thickness) was $21 \cdot 34 \pm 4 \cdot 49$ in the 4-12-year-old group and was $35 \cdot 86 \pm 7 \cdot 12$ in the 13-20-year-old group, which showed the significant difference $(P<0 \cdot 01)$. The difference was also significant between the 4-12-year-old group $(27 \cdot 81 \pm 4 \cdot 14)$ and the 13-20-year-old group $(37 \cdot 83 \pm 7 \cdot 8)$ in female adrenals $(P<0 \cdot 01$; Fig. $1 \mathrm{C})$.

\section{Comparison between male and female adrenals}

There were no significant sex differences in the total adrenal thickness among any of the three age groups studied $(P>0 \cdot 05)$. In addition, adrenals from neither the 4-12year-old group nor the 13-20-year-old group demonstrated a significant sex difference in the thickness of the ZR and the $R$ values $(P>0 \cdot 05)$.

\section{Immunohistochemistry for KI67}

KI67-MIB1 (Goldblum et al. 1993, Sasano et al. 1995) immunoreactivity was detected only in the nuclei of adrenocortical cells but not in those of capsule and medulla. In male adrenals, the immunoreactivity in the 0-3-year-old group was focally present in zona glomerulosa $(\mathrm{ZG})$ with the highest LI (\%) of $6 \cdot 6$ at 8 months of age and the average LI was $5 \cdot 22 \pm 1 \cdot 19$; KI67-positive cells appeared in the ZR at 4 years of age and its number reached a peak at 12 years of age with the LI of $13 \cdot 0$. The average KI67-LI in the 4-12-year-old group was $5 \cdot 63 \pm 3 \cdot 40$; KI67 immunoreactivity in ZR progressively declined around 20 years of age with the average KI67-LI of $2.64 \pm 1.50$ in the 13-20-year-old group. Regardless of its intra-adrenal location of immunoreactivity, KI67-LI was higher in the 4-12-year-old group than that seen in the $13-20$-year-old group $(P<0 \cdot 05)$. In female adrenals, immunoreactivity in specimens younger than 4 years of age was also mainly located in the ZG or sometimes in the outer ZF with the LI $(\%)$ of $7 \cdot 05 \pm 3 \cdot 10$ in the $0-3$-year-old group and the highest LI of $10 \cdot 2$ was detected at 7 months of age. However, the KI67-positive adrenocortical cells were also present in ZF before becoming discernible in ZR around 4 or 5 years of age and then increased later. The highest KI67-LI of 11.5 was detected predominantly in the inner ZR and partly in ZF at 15 years of age followed by progressive decrement toward 20 years of age (LI in the 4-12-year-old group was 2.50 $\pm 1 \cdot 44$; LI in the 13-20-year-old group was $4 \cdot 28 \pm 3 \cdot 62)$. The representative images of distribution of KI67 immunoreactivity were summarized (Fig. 2A and B). The levels of KI67-LI in these three age groups are summarized in Table 1.

\section{Immunohistochemistry for BCL2}

Previous studies reported the expression of BCL2 in normal human adrenal cortex (Fogt et al. 1998, Bernini et al. 2002).
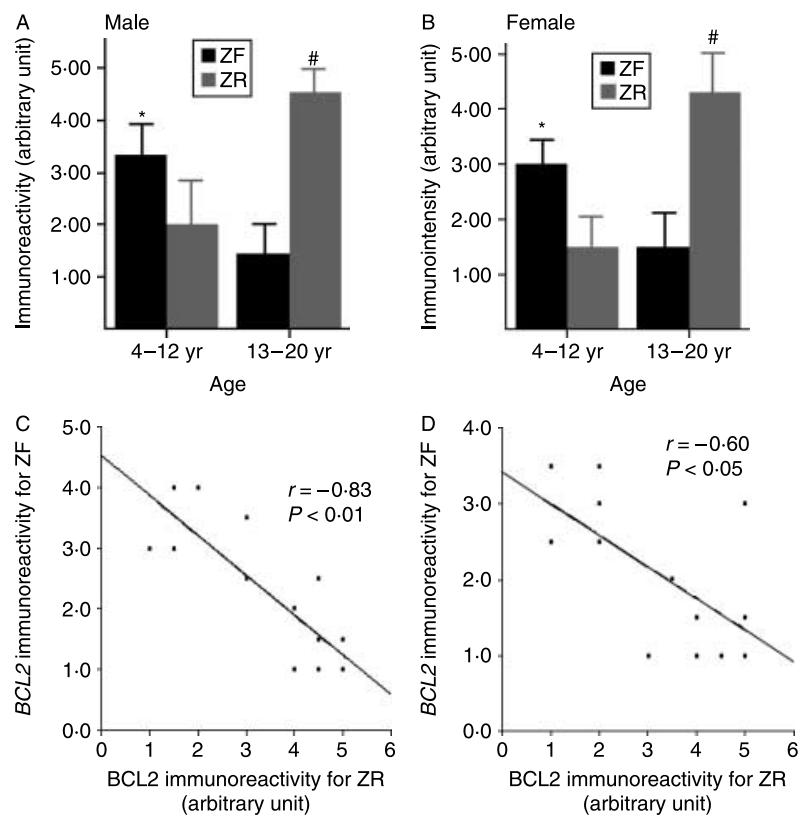

Figure 3 The comparisons of immunoreactivity of $B C L 2$ between the ZF and the ZR in human young adrenals. The higher expression of $B C L 2$ was demonstrated in the $Z F$ in 4-12 year olds but in the $Z R$ in $13-20$ year olds of both male $\left((A) *\right.$ versus $Z R ;{ }^{\#}$ versus $\left.Z F, P<0 \cdot 01\right)$ and female adrenals $\left((B){ }^{*}\right.$ versus $Z R ;{ }^{*}$ versus $\left.Z F, P<0 \cdot 01\right)$. Besides, the inversed correlation of $B C L 2$ immunoreactivity between the $Z R$ and the ZF was demonstrated in both male $(r=-0 \cdot 83, P<0 \cdot 01, \mathrm{C})$ and female adrenals $(r=-0 \cdot 50, P<0 \cdot 05, \mathrm{D})$. 

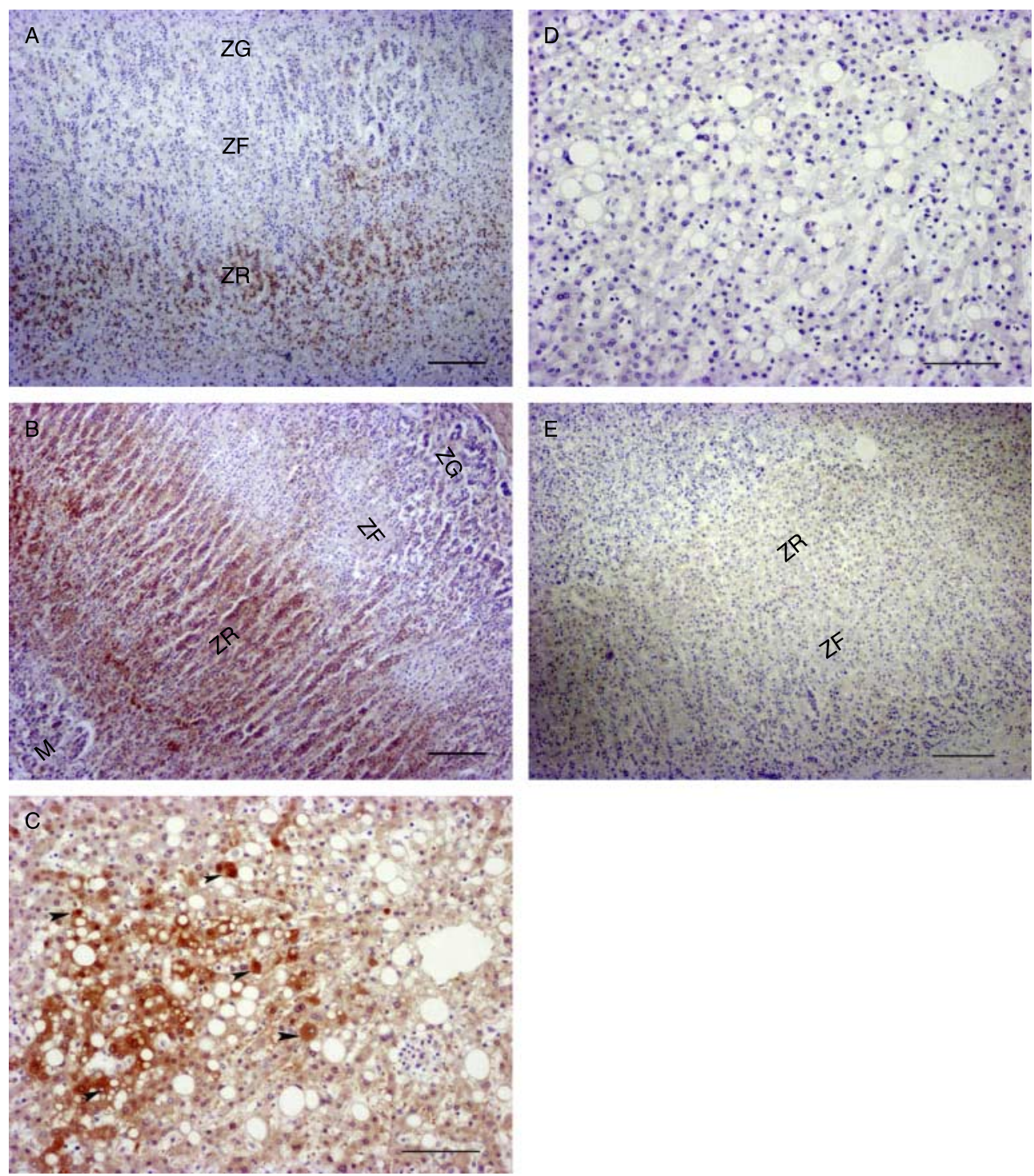

Figure 4 Immunoreactivity of HSD17B5 in young human adrenals. As shown in the representative micrograph, the immunoreactivity of HSD17B5 was not detected in the cytoplasm of adrenocortical cells in the ZR until around 9 years of age (A), followed by increased expression in the ZR at 19 years of age (B). Positive control for immunohistochemistry was demonstrated in human liver paraffin-embedded section (C, see arrowhead); negative controls were illustrated in human liver and adrenal gland specimens respectively ( $D$ and $E)$. Bar represents $200 \mu \mathrm{m}(A, B$, and $E)$; bar represents $100 \mu \mathrm{m}(C$ and $D)$. ZG, zona glomerulosa; ZF, zona fasciculata; $Z R$, zona reticularis; $M$, medulla. Full colour version of this figure available via http://dx.doi.org/10.1677/JOE-09-0127.

In our present study, BCL2 immunoreactivity was also demonstrated in the cytoplasm of adrenocortical cells but not in medulla or capsule. The age-related changes of BCL2 immunoreactivity were summarized in Table 2. Briefly, before 4 years of age, relatively lower expression of $B C L 2$ was detected in the adrenal specimens (it was recorded as 0 due to the absence of ZR). After 4 years of age, BCL2-positive cells frequently appeared in the ZF, especially in the outer part of this zone. In addition, BCL2 immunoreactivity in the $\mathrm{ZF}$, mainly in the inner ZF adjacent to the ZR, decreased while it increased in the $\mathrm{ZR}$ with ages (Fig. $2 \mathrm{C}$ and D). Its immunoreactivity was significantly higher in the $\mathrm{ZF}$ than in the $\mathrm{ZR}$ in the 4-12-year-old group but higher in the $\mathrm{ZR}$ than in the ZF in the 13-20-year-old group (Fig. 3A and B). The inversed correlation of immunoreactivity between the $\mathrm{ZF}$ and the ZR was also demonstrated in both male and female adrenals $(r=-0 \cdot 827, P<0 \cdot 01$ in male; $r=-0 \cdot 595$, $P<0 \cdot 05$ in female; Fig. $3 \mathrm{C}$ and D). 


\section{Immunohistochemistry for HSD17B5}

HSD17B5 immunoreactivity was detected in the cytoplasm of the ZR but not in the medulla or other cortical zones. In both male and female adrenals, HSD17B5 immunoreactivity in $\mathrm{ZR}$ was not easily discernible until $\sim 9$ years of age, followed by increment with age up to 20 years of age (Fig. $4 \mathrm{~A}$ and $\mathrm{B})$. The regression analysis demonstrated a significant positive correlation between the morphologically determined thickness of the ZR and that of HSD17B5 immunoreactive $\mathrm{ZR}(r=0.991, P<0.01$ in male; $r=0.989, P<0 \cdot 01$ in female; Fig. 5A and B).
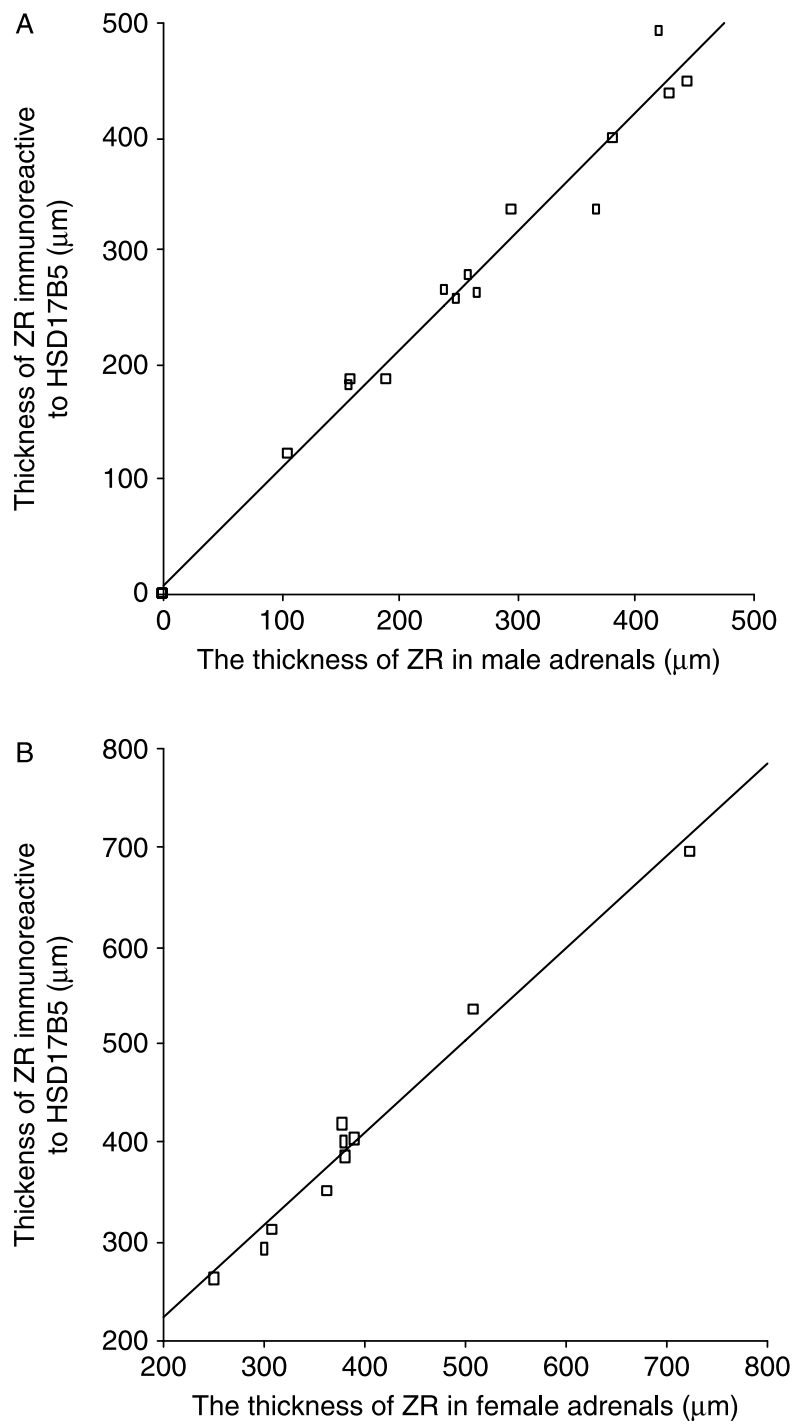

Figure 5 Analysis of correlation revealed that the morphologically identifiable ZR thickness corresponded well to the thickness of the ZR that was immunopositive for HSD17B5 in both male $((\mathrm{A}) r=0.99, P<0 \cdot 01)$ and female adrenals $((\mathrm{B})$ $r=0.99, P<0 \cdot 01)$.

\section{Discussion}

Adrenal DHEAS secretion, in general, demonstrates a unique age-dependent pattern during the human life span. Before birth, DHEAS rises progressively to reach a peak at term followed by precipitous decline during the first months of life and remains low until adrenarche commences at $\sim 6-8$ years of age (Deperetti \& Forest 1976). Serum DHEAS levels continue to rise and reach a peak during the second decade of life (Orentreich et al. 1984). The production of DHEAS is so enormous during certain periods of our life span that DHEAS is indeed quantitatively the most abundant steroid hormone secreted by human adrenal. In addition, the ZR thickness has, in general, been postulated to correspond to increased production of DHEA and DHEAS (Dhom 1973, Reiter et al. 1977). It is, however, nearly impossible to correlate an increment in individual DHEAS levels during adrenarche with corresponding morphology of the ZR even employing sophisticated image analyses, and the functional development of the ZR has not been reported. Therefore, in our present study, we quantitatively and chronologically examined ZR development in young human adrenals using autopsy materials. Results of our quantitative study demonstrated the followings: 1) the total thickness of the adrenal cortex was significantly greater in older age groups, possibly due to the near absence of the adrenal ZR in younger children; 2) after 4 years of age, the expansion of the ZR started but did not result in a significant increment in the total thickness of the cortex, suggesting that the early stages of adrenarche relate to intra-adrenal remodeling (Figs 6 and 7).

Classically, human adrenarche was postulated to start between 6 and 8 years of age. Dhom (1973) reported that a continuous ZR development started to be seen around 6 years of age, which is different from 4 years of age, suggested in our study. However, results of our study are similar to more recent studies suggesting the morphologic onset of ZR probably occurs around 4 years of age (Marx et al. 1997, Suzuki et al. 2000). This discrepancy may be related to employment of immunohistochemistry to localize $\mathrm{ZR}$ in addition to histological recognition in the later studies. Results of our study, which clearly demonstrated the morphologic appearance of functional ZR cells before 6 years of age, are also in agreement with the reports by Remer and coworkers (Remer et al. 1994) showing earlier detection of urinary adrenal androgens. Results of these morphologic and steroid studies all suggest that the gradual onset of adrenarche occurs earlier than previously postulated, but further investigations are required for clarification.

In the present study, we also demonstrated subtle differences for the age-related progressive development of the ZR between male and female adrenals. Before the onset of ZR development, the total adrenal cortex in female was thicker than that of males within the earlier years of life. Following the onset of puberty, both the total width and the ZR thickness of female adrenals exhibited a faster 

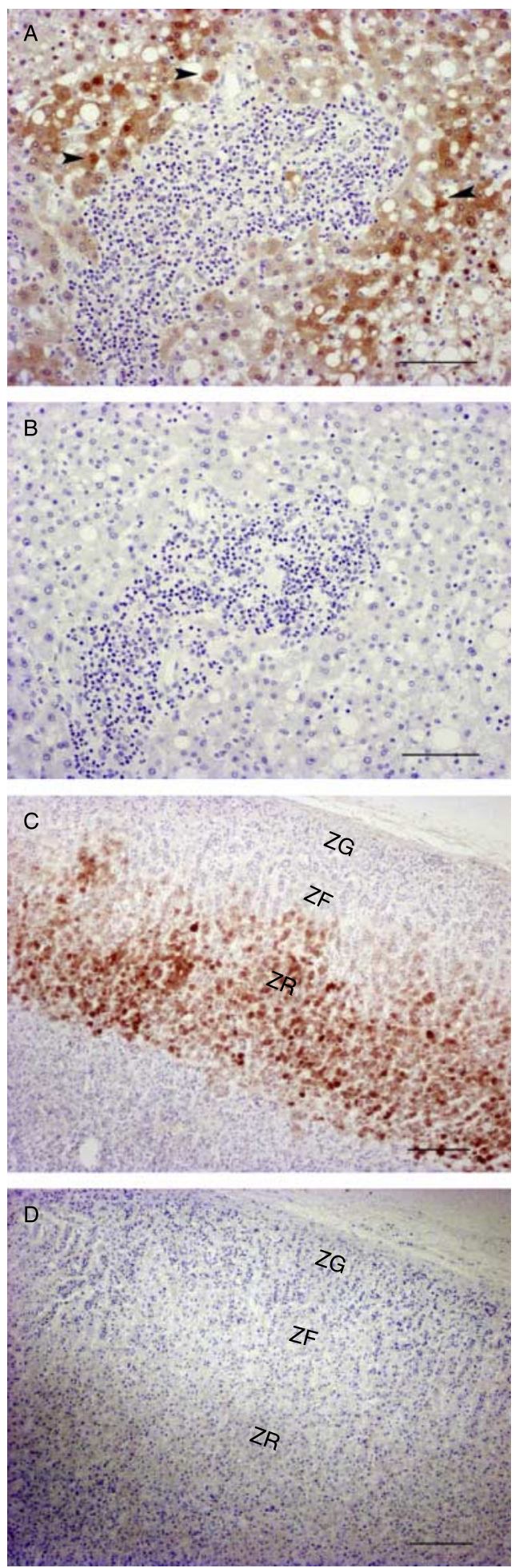

www.endocrinology-journals.org development than in male adrenals. In particular, the ratio of $\mathrm{ZR}$ thickness of the female adrenal around 16 or 17 years of age in the 13-20-year-old group surpassed $50 \%$ of the total cortex suggestive of marked development or remodeling particularly in female adrenals, though there were no significant gender differences in total cortex thickness during this period of development. Deperetti \& Forest (1976) demonstrated a trend for higher levels of circulating DHEA in girls than in boys from 6 to 10 years of age, but the differences did not reach statistical significance. Therefore, we could not draw a definitive conclusion regarding the sex difference in human adrenals, but female adrenals tended to demonstrate a relatively rapid development of $\mathrm{ZR}$. It awaits further investigations for clarification.

The temporal and spatial intra-adrenal patterns of KI67positive adrenocortical cells are not only consistent with a putative characteristic of centripetal migration, but also with the hypothesis that the multipotential progenitor cells may exist in the outer $\mathrm{ZF}$ or $\mathrm{ZG}$ in human adrenal cortex. Lymphocytes and macrophages became discernible in human adrenals near 20 years of age, and the expression of KI67 in the adrenocortical parenchymal cells accordingly declined in these age groups suggesting that the apoptotic ZR cells were possibly removed by phagocytosis, which consequently provide space for the centripetal replacement of ZR cells. Macrophages are detected in the inner zone and belong to the phagocytic compartment characterized by the expression of KiM8 and the adhesion molecules CD68 (GonzaiezHernandez et al. 1994). They may serve as the scavengers of the apoptotic remnants in the inner cortical layer (Wyllie et al. 1980, Nathan 1987, Hayashi et al. 1989), which may increase in number after 20 years of age.

BCL2 encodes proteins that inhibit apoptosis, thus prolonging the survival of cell populations (Hockenbery et al. 1990). Results of our present study demonstrated that BCL2 expression was frequently detected in the outer ZF and inversely correlated with its expression in $\mathrm{ZR}$. Therefore, the outer ZF may actively inhibit apoptosis, and the maintenance of the ZR may be achieved by allowing apoptosis or functional conversion of ZR cells at the inner ZF cells or reticularis/fasciculata border. Parker (1991) demonstrated that after ACTH treatment, the compact ZR widens, sometimes sufficiently to encroach on the adjacent ZF. However, it is also important to note that negative expression of BCL2 does not

Figure 6 The validation of immunohistochemistry for the antibody against SULT2A1. (A) Immunohistochemical detection in human liver paraffin-embedded section as a positive control of immunostaining (see arrowhead); (B) no immunoreactivity was detected in human liver paraffin-embedded section where primary antibody was omitted; (C) marked immunoreactivity was detected in the ZR of human adrenal gland; (D) no specific immunoreactivity was detected in human adrenal gland where the primary antibody was omitted. Bar represents $100 \mu \mathrm{m}$ (A and B); bar represents $200 \mu \mathrm{m}$ (C and D). ZG, zona glomerulosa; ZF, zona fasciculata; ZR, zona reticularis. Full colour version of this figure available via http://dx.doi.org/10.1677/JOE-09-0127. 

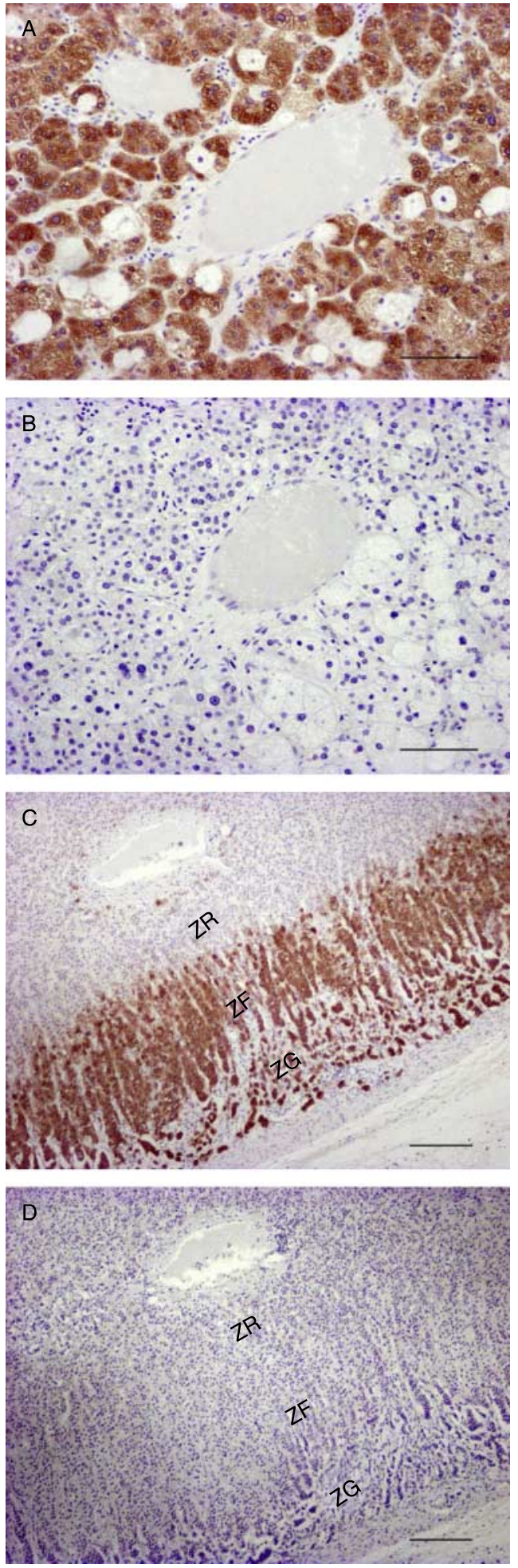

Journal of Endocrinology (2009) 203, 241-252 necessarily represent the occurrence of apoptosis as terminal deoxynucleotidyl transferase-mediated dUTP nick end labeling (TUNEL) assay. TUNEL assay could not be performed in our series because of the nature of the materials obtained from autopsy. Therefore, it also awaits further investigations for clarification.

Results of KI67 and BCL2 in developing human adrenals in our present study are consistent with the classical theory of zonation driven by cell migration in human adrenal glands, which was also proposed by Sasano et al. (1995). In the migration model, each zone is derived from a common pool of progenitor cells located in the peripheral cortex, which then migrate inward and differentiate to populate the inner cortical zones (Sugihara et al. 1977, Gottschau 1983), while the $\mathrm{ZR}$ is considered a zone of cell senescence followed by their loss from the system following apoptosis (Hoerr 1931, Nussdorfer 1986). Results of our present study also demonstrated that proliferative adrenocortical cells invariably exist in outer cortex before 4 years of age followed by abundant expression in ZR during human pubertal ages. The centripetal stream of proliferative parenchymal cells throughout the development may contribute to the enlargement of the ZR. In addition, the balance between KI67 and BCL2 is also considered as the key regulators controlling the development and maturation of $\mathrm{ZR}$ in human adrenal glands.

The onset of puberty overlaps with adrenarche and ZR development, but the correlation between these two processes is unclear. Adrenarche has been hypothesized to have an effect on $\mathrm{GnRH}$ secretion and the pubertal process because of the increase in adrenal androgen biosynthesis that occurs prior to puberty (Ducharme et al. 1976). However, DHEA and DHEAS are relatively poor regulators of the androgen receptor and their ability to influence the hypothalamus is not clear. Very recently, Nakamura et al. (2009) have demonstrated that ZR cells express HSD17B5 in human adult adrenals and confirmed that ACTH administration increased serum testosterone levels in female adrenal vein samples, suggesting that HSD17B5 in ZR may play an important role in the production of testosterone in human adrenals. The production of more potent androgens, even in low levels, during the course of adrenarche could provide a new link between adrenarche and puberty. Such androgens would also be more likely to control the process of pubarche, which is considered to occur as a result of adrenarche. In our

Figure 7 The validation of immunohistochemistry for the antibody against HSD3B2. (A) Immunoreactivity was markedly present in the cytoplasm of tumor cells in human Cushing's adenoma employed as positive control of immunostaining; (B) no immunoreactivity was detected in human Cushing's adenoma where primary antibody was omitted; (C) marked immunoreactivity was detected in the adrenocortical cells of ZG and ZF of human normal adrenal gland; (D) no immunoreactivity was detected in human normal adrenal gland where primary antibody was omitted. Bar represents $100 \mu \mathrm{m}$ (A and B); bar represents $200 \mu \mathrm{m}$ (C and D). ZG, zona glomerulosa; $\mathrm{ZF}$, zona fasciculata; ZR, zona reticularis. Full colour version of this figure available via http://dx.doi.org/10.1677/JOE-09-0127. 
present study, HSD17B5 was predominantly detected in the ZR of human adrenals around 9 years of age when the pubertal process is considered to be initiated. In addition, some boys with poor adrenal androgen suppression due to undertreated congenital adrenal hyperplasia developed true precocious puberty (Boyar et al. 1973). Therefore, adrenal HSD17B5 may be involved in pubarche and the onset of puberty by producing more active androgens. Results of our present study do not necessarily establish a definitive correlation between puberty and ZR development, but could provide further insights into the temporal association of the two processes.

\section{Declaration of interest}

The authors declare that there is no conflict of interest that would prejudice the impartiality of the research reported.

\section{Funding}

This work was supported by grants from the National Institutes of Health (DK069950 and DK43140), a grant for Research on Intractable Diseases (H20-intractability-009), and a grant for Risk Analysis Research on Food and Pharmaceuticals (H19-Chemicals-004) from Ministry of Health, Labour, and Welfare, Japan.

\section{References}

Bernini GP, Moretti A \& Viacava P 2002 Apoptosis control and proliferation marker in human normal and neoplastic adrenocortical tissues. British Journal of Cancer 86 1561-1565.

Boyar RW, Finkelstein JW, David R \& Roffwarg H 1973 Twenty-four h patterns of plasma luteinizing hormone and follicle-stimulating hormone in sexual precocity. New England Journal of Medicine 289 282-286.

Comer KA, Falany JL \& Falany CN 1993 Cloning and expression of human liver dehydroepiandrosterone sulfotransferase. Biochemical Journal 289 233-240.

Dardis A, Saraco N, Rivarola MA \& Belgorosky A 1999 Decrease in the expression of the $3 \beta$-hydroxysteroid dehydrogenase gene in human adrenal tissue during prepuberty and early puberty: implications for the mechanism of adrenarche. Pediatric Research 45 384-388.

DePeretti E \& Forest MG 1976 Unconjugated dehydroepiandrosterone plasma levels in normal subjects from birth to adolescence in human: the use of a sensitive radioimmunoassay. Journal of Clinical Endocrinology and Metabolism 43 982-991.

Dharia S, Slane A, Jian M, Conner M, Conley AJ, Brissie RM \& Parker CR 2005 Effects of aging on cytochrome b5 expression in the human adrenal gland. Journal of Clinical Endocrinology and Metabolism 90 4357-4361.

Dhom G 1973 The prepberal and puberal growth of the adrenal (adrenarche). Beiträge zur Pathologie 150 357-377.

Doody KM, Carr BR \& Rainey WE 1990 3ß-Hydroxysteroid dehydrogenase/isomerase in the fetal zone and neocortex of the human fetal adrenal gland. Endocrinology 126 2487-2492.

Ducharme JR, Forest MG, Deperetti E, Sempe M, Collu R \& Bertrand J 1976 Plasma adrenal and gonadal sex steroids in human pubertal development. Journal of Clinical Endocrinology and Metabolism 42 468-476.

Dufort I, Rheault P, Huang XF, Soucy P \& Luu-The V 1999 Characteristics of a highly labile human type $517 \beta$-hydroxysteroid dehydrogenase. Endocrinology 140 568-574.

Endoh A, Kristiansen SB, Casson PR, Buster JE \& Hornsby PJ 1996 The zona reticularis is the site of biosynthesis of dehydroepiandrosterone and dehydroepiandrosterone sulfate in the adult human adrenal cortex resulting from its low expression of $3 \beta$-hydroxysteroid dehydrogenase. Journal of Clinical Endocrinology and Metabolism 81 3558-3565.

Fogt F, Vortmeyer AO, Poremba C, Minda M, Harris CA \& Tomaszewski JE $1998 \mathrm{Bcl}-2$ expression in normal adrenal glands and in adrenal neoplasms. Modern Pathology 11 716-720.

Forbes KJ, Hagen M, Glatt H, Hume R \& Coughtrie WH 1995 Human fetal adrenal hydroxysteroid sulphotransferase: cDNA cloning, stable expression in V79 cells and functional characterisation of the expressed enzyme. Molecular and Cellular Endocrinology 112 53-60.

Geissler WM, Davis DL, Wu L, Bradshaw KD, Patel S, Mendonca BB, Elliston KO, Wilson JD, Russell DW \& Andersson S 1994 Male pseudohermaphroditism caused by mutation of testicular 17beta-hydroxysteroid dehydrogenase 3. Nature Genetics 7 34-39.

Gell JS, Atkins B, Margraf L, Mason JI, Sasano H \& Rainey WE 1996 Adrenarche is associated with decreased $3 \beta$-hydroxysteroid dehydrogenase expression in the adrenal reticularis. Endocrine Research 22 723-728.

Gell JS, Carr BR, Sasano H, Atkins B, Margraf L \& Mason JI 1998 Adrenarche results from development of a $3 \beta$-hydroxysteroid dehydrogenase-deficient adrenal reticularis. Journal of Clinical Endocrinology and Metabolism 83 3695-3701.

Goldblum J, Shannon R, Kaldjian EP, Thiny M, Davenport R, Thompson N \& Lloyd R 1993 Immunohistochemical assessment of proliferative activity in adrenal cortical neoplasms. Modern Pathology 6 663-668.

Gonzaiez-Hernandez JA, Bornslein SR, Ehrhart-Bornslein M \& Gschwend JE 1994 The macrophages within the human adrenal gland. Cell and Tissue Research 278 201-205.

Gottschau M 1983 Struktur and embyonale dennebenniere bei Saugetieren. Archiv fur Anatomie und Entuecklungsgeschichte. Anatomie Abteilung 9 412-457.

Hayashi Y, Hiyoshi T, Takemura T, Kurashima C \& Hirokawa K 1989 Focal lymphocytic infiltration in the adrenal cortex of the elderly: immunohistochemical analysis of infiltrationg lymphocytes. Clinical and Experimental Immunology 77 101-105.

Hockenbery DM, Nunez G, Milliman C, Schreiber RD \& Korsmeyer SJ $1990 \mathrm{Bcl}-2$ is an inner mitochondrial membrane that blocks programmed cell death. Nature 348 334-337.

Hoerr NL 1931 The cells of the suprarenal cortex in the guinea pig. American Journal of Anatomy 48 139-197.

Hyatt PJ, Bhatt K \& Tait GF 1983 Steroid biosynthesis by zona fasciculata and zona reticularis cells purified from the mammalian adrenal cortex. Journal of Steroid Biochemistry 19 953-960.

Labrie F, Luu-The V, Belanger A, Lin SX, Simard J, Pelletier G \& Labrie C 2005 Is dehydroepiandrosterone a hormone? Journal of Endocrinology 187 169-196.

Leblond CP 1964 Classification of cell populations on the basis of their proliferative behavior. National Cancer Institute Monograph 14 119-150.

Lin HK, Steckelbroeck S, Fung KM, Jones AN \& Penning TM 2004 Characterization of a monoclonal antibody for human aldo-keto reductase AKR1C3 (type 2 3alpha-hydroxysteroid dehydrogenase/type 5 17betahydroxysteroid dehydrogenase); immunohistochemical detection in breast and prostate. Steroids $\mathbf{6 9} 795-801$.

Lorence MC, Murry BA, Trant JM \& Mason JI 1990 Human 3ßhydroxysteroid dehydrogenase $/ \mathrm{D}^{5 \rightarrow 4}$ isomerase from placenta: expression in nonsteroidogenic cells of a protein that catalyzed the dehydrogenation/isomerization of C21-C19 steroids. Endocrinology 126 2493-2498.

Magrini U, Bertoli G \& Mazzola R 1969 Histochemistry of 3ßhydroxysteroid dehydrogenase in human adrenal cortex in various pathological conditions. Pathologia Europaea 4 30-41.

Mapes S, Corbin J, Tarantal A \& Conley A 1999 The primate adrenal zona reticularis is defined by expression of cytochrome b5, 17 $\alpha$-hydroxylase/17,20-lyase cytochrome p450 (p450c17) and NADPH-cytochrome $\mathrm{p} 450$ reductase (reductase) but not $3 \beta$-hydroxysteroid dehydrogenase $/ \Delta 5-4$ isomerase (3ß-HSD). Journal of Clinical Endocrinology and Metabolism 84 $3382-3385$ 
Martinez-Valdez H, Guret C, de Bouteiller O, Fugier I, Banchereau J \& Liu YJ 1996 Human germinal center B cells express the apoptosisinducing genes Fas, c-myc, $\mathrm{p}^{53}$, and Bax but not he survival gene bcl-2. Journal of Experimental Medicine 183 971-977.

Marx C, Borustein SR, Wokiersdorfer GW, Peter M, Sippell WG \& Scherbaum WA 1997 Relevance of major histocompatibility complex class $\alpha$ expression as a hallmark for the cellular differentiation in the human adrenal cortex. Journal of Clinical Endocrinology and Metabolism 82 3136-3140.

McNicol AM 1992 The human adrenal gland: aspect of structure, function and pathology. In The Adrenal Gland, pp 1-42. Ed. VHT James. New York: Raven Press.

Nakamura Y, Hornsby PJ, Casson P, Morimoto R, Satoh F, Xing Y, Kennedy MR, Sasano H \& Rainey WE 2009 Type 5 17ß-hydroxysteroid dehydrogenase (AKR1C3) contributes to testosterone production in adrenal reticularis. Journal of Clinical Endocrinology and Metabolism 94 2192-2198.

Narasaka T, Moriya T, Endoh M, Suzuki T, Shizawa S, Mizokami Y, Matsuoka T \& Sasano H 2000 17ß-Hydroxysteroid dehydrogenase type 2 and dehydroepiandrosterone sulfotransferase in the human liver. Endocrine Journal 47 697-705.

Nathan CF 1987 Secretory products of macrophages. Journal of Clinical Investigation 79 319-326.

Nussdorfer GG 1986 Cytophysiology of the adrenal cortex. International Review of Cytology 98 1-405.

Orentreich N, Brind JL, Rizer RL \& Vogelman JH 1984 Age changes and sex differences in serum dehydroepiandrosterone sulphate concentrations throughout adulthood. Journal of Clinical Endocrinology and Metabolism 59 551-555.

Otterness DM, Wieben ED, Wood TC, Watson RWG, Madden BJ, McCormick DJ \& Weinshilboum RM 1992 Human liver dehydroepiandrosterone sulfotransferase: molecular cloning and expression of cDNA. Molecular Pharmacology 41 865-872.

Parker LN 1991 Adrenarche. Endocrinology and Metabolism Clinics of North America 20 71-83.

Parker LN \& Odell WD 1980 Control of adrenal androgen section. Endocrine Reviews 1 392-410.

Parker LN, Lifrak ET, Ramadan MB \& Lai MK 1983 Aging and the human zona reticularis. Archives of Andrology 10 17-20.

Parker CR Jr, Mixon RL, Brissie RM \& Grizzle WE 1997 Aging alters zonation in the adrenal cortex of men. Journal of Clinical Endocrinology and Metabolism 82 3898-3901.

Petry CJ, Ong KK, Wingate DL, de Zegher F, Ibáñez L \& Dunger DB 2007 Lack of association between common polymorphisms in the 17betahydroxysteroid dehydrogenase type V gene (HSD17B5) and precocious pubarche. Journal of Steroid Biochemistry and Molecular Biology 105 176-180.
Qin KN \& Rosenfield RL 2000 Expression of 17 beta-hydroxysteroid dehydrogenase type 5 in human ovary: a pilot study. Journal of the Society for Gynecologic Investigation 7 61-64.

Rainey WE \& Nakamura Y 2008 Regulation of the adrenal androgen biosynthesis. Journal of Steroid Biochemistry and Molecular Biology 108 281-286.

Rainey WE, Carr BR, Sasano H, Suzuki T \& Mason JI 2002 Dissecting human adrenal androgen production. Trends in Endocrinology and Metabolism $13234-239$.

Reiter EO, Fuldauer VG \& Root AW 1977 Secretion of the adrenal androgens dehydroepiandrosterone sulfate during normal infancy, childhood, and adolescence in sick children and in children with endocrinologic abnormalities. Journal of Pediatrics 90 766-770.

Remer T, Hintelmann A \& Manz F 1994 Measurement of urinary androgen sulfates without previous hydrolysis: a tool to investigate adrenarche. Determination of total 17-ketosteroid sulfates. Steroids 59 16-21.

Sasano H, Mason JI, Sasano N \& Nagura H 1990 Immunolocalization of 3ßhydroxysteroid dehydrogenase in human adrenal cortex and in its disorders. Endocrine Pathology 1 94-101.

Sasano H, Imatani A, Shizawa S, Suzuki T \& Nagura H 1995 Cell proliferation and apoptosis in normal and pathologic human adrenal. Modern Pathology 8 11-17.

Sugihara H, Kawai K \& Tsuchiyama H 1977 Effects of exogenous ACTH on the rat fetal adrenal cortex: a histochemical and electron-microscopical observation. Acta Pathologica Japonica 27 477-484.

Suzuki T, Sasano H, Takeyama J, Kaneko C, Freije WA, Carr BR \& Rainey WE 2000 Development changes in steroidgenic enzymes in human postnatal adrenal cortex: immunohistochemical studies. Clinical Endocrinology 53 739-747.

Voutilainen R, Perheentupa J \& Apter D 1983 Benign premature adrenarche: clinical features and serum steroid levels. Acta Paediatrica Scandinavica 72 707-711.

Wyllie AH, Kerr JFR \& Currie AR 1980 Cell death: the significance of apoptosis. International Review of Cytology 68251.

Yanase T, Sasano H, Yubisui T, Sakai Y, Takayanagi R \& Nawata H 1998 Immunohistochemical study of cytochrome b5 in human adrenal gland and in adrenocortical adenomas from patients with Cushing's syndrome. Endocrine Journal 45 89-95.

Received in final form 3 August 2009

Accepted 1 September 2009

Made available online as an Accepted Preprint 1 September 2009 